\title{
Droplet interaction and dynamic wettability of advanced materials used in aeronautics
}

\author{
O. Gohardani \& D. W. Hammond \\ Department of Power and Propulsion, Cranfield University, UK
}

\begin{abstract}
In aircraft icing applications the interaction of Super-Cooled Liquid Droplets (SLD) with the forward facing surfaces of an aircraft is of outmost importance, as it influences the consequent icing that ensues on the structure. In this study, empirical results are shown that characterize different droplet splashing scenarios onto polymeric matrix composites reinforced with carbon nanotubes. Results are presented for droplet interaction upon advanced pristine and eroded aerospace materials based on a numerical scheme, that identifies different splashing features into distinct categories. The purpose of utilizing the dynamic wetting analysis scheme is to numerically represent the type of splashing events that are evident on the experimental photographs. Images are presented for splashing features on different surfaces and their similarities and differences are discussed based on the analysis. Hydrophobicity and hydrophilicity of the different specimen surfaces are further examined based on both static and dynamic contact angle measurements and a correlation between the two modes of wetting is presented for the aforementioned materials.

Keywords: dynamic wettability, contact angle, droplet interaction, carbon nanotubes, aeronautics.
\end{abstract}

\section{Introduction}

The potential usage of advanced materials such as polymeric matrix composites and carbon nanotubes have widely been recognized due to the number of possible applications where these materials may present desired characteristics. In aerospace applications, composite materials are widely being considered because of their higher stiffness and strength [1]. The present study investigates the 
interaction of droplets on advanced materials and the resulting splashing that occurs empirically. This interaction is comparable to the splashing that occurs upon the airframe of an aircraft, flying through a cloud formation or when subjected to precipitation. Weathering effects due to extended service operations of aircraft in flight may occasionally result in structural wear on the airframe and erosion as described in an extensive review by Gohardani [2]. For this purpose the considered specimens have been modified by erosion, in order to investigate the role of surface morphology on the ensuing splashing structures.

Wetting characteristics outside aerospace sciences have been examined in a wide range of applications, where in particular the role of wetting on micro and nanostructures in biological applications, has been studied for plant surfaces [3], insect wing structures [4] and animal locomotion on the surface of water layers [5].

\section{Experimental details}

\subsection{Experimental setup}

The experiments in this study are carried out at the vertical droplet tunnel located at Cranfield University, United Kingdom. The tunnel is located next to the main Icing Tunnel, with the flow in both tunnels powered by a centrifugal backward curved suction fan, capable of producing flow rates, $\dot{m} \in[30,100] \mathrm{kg} \cdot \mathrm{s}^{-1}$. The temperature range $T \in[-30,+5]^{\circ} \mathrm{C}$ is attained by the refrigeration plant which has a capacity of $400 \mathrm{~kW}$. Upon generation of the flow, the air from the fan is directed through a duct into a heat exchanger for cooling and directed into a steering dish in the adjacent vertical tunnel where it gets accelerated toward the test section. A droplet generator equipped with an interchangeable platinum nozzle orifice disk is placed on top of the steering dish.

Upon entering the contraction section of the vertical droplet tunnel, the mono dispersed droplets are accelerated towards the test section, where the gentle contraction length of 5 meters, ensures that no aerodynamic breakup of the droplets occurs. The test section is situated on top of two control valves, capable of regulating the locus of the stagnation point. The target specimen is placed on a designed target holder, that allows for vertical position adjustment of the specimen. This is essential in order to account for the range of different specimen thicknesses within the study. The incident angle of the oncoming air in relation to the target is $\alpha=70^{\circ}$. Upon contact with the target area the flow is bifurcated and exits the tunnel through the control valves into the air inlet of the main Icing Tunnel, where it gets regenerated by the fan. The droplet diameter in this study has been confined to $d=400 \mu \mathrm{m}$, with three different free stream velocities $U_{\infty} \approx\{35,50,60\}$ $\mathrm{m} \cdot \mathrm{s}^{-1}$, in accordance with expected number of splashing structures for statistical purposes. The mono dispersed droplets are ejected from the orifice of the droplet generator at a frequency of $f_{d} \approx 12 \mathrm{kHz}$. Figure 1 shows the experimental setup for the dynamic wettability experiments.

The illumination of the target area is attained by directing the light from an LED through a collimating lens system. The LED device is strobed with a pulse 


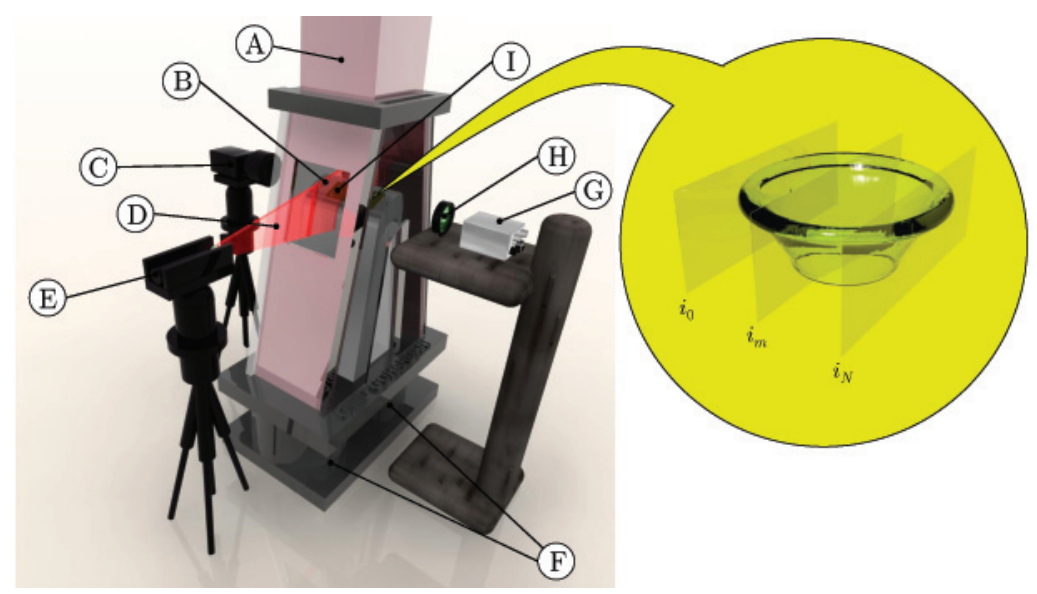

Figure 1: The experimental setup for the dynamic wettability experiments with notations: (A) Vertical droplet tunnel, (B) droplet cloud, (C) CCD camera, (D) laser sheet, (E) laser, (F) control valves, (G) LED strobing device, $(\mathrm{H})$ collimating lens, and (I) specimen target. The right hand side image depicts a schematic corona structure with virtual planes $i_{0}, \ldots, i_{N}$, with $i_{m}$ showing the mid-plane.

period $T=40 \mathrm{~ms}$ and pulse length of $\tau=5 \mu$ s which results in approximately one strobe per acquired frame of the experiment. The image acquisition is actualized by employing a Sony XCD-SX90 camera operated at 30 FPS. The camera is equipped with a $50 \mathrm{~mm}$ lens and $13 \mathrm{~mm}$ extension. The distance between the camera and the wall of tunnel $d_{W}=50 \mathrm{~mm}$, the LED and the collimating lens $d_{C}=40 \mathrm{~mm}$, and further between the collimating lens and the wall of the tunnel is $100 \mathrm{~mm}$.

The optical magnification of the experimental image is hence 0.32 . The images are acquired upon utilizing a graphical user interface on a personal computer.

\subsubsection{Integrated view}

As the LED light is captured along the length of the specimens, splashing features are apparent in the volume of space along the width of each specimen. The image acquisition camera is focused on the mid-plane of the specimen surface as denoted by $i_{m}$ in Figure 1. This entails that any structure appearing within this plane of sight will appear entirely in focus on the experimental photographs. Equally, a deviation from this plane will result in structures appearing not entirely in focus. However, as the employed illumination within the depth of field is adequate, even off-plane splashing features are distinguishable. This approach hence results in capturing of most splashing events, at the expense of them appearing simultaneously on similar locations along the width of the specimen which may result in distinction of certain features becoming intricate. It is notable 
that as the LED light travels through all virtual planes $i_{0}, \ldots, i_{N}$, an integrated view is visible upon image acquisition.

\subsubsection{Planar view}

In order to estimate the local liquid water content on the specimen surface, a laser sheet with a $2 \mathrm{~mm}$ width was used to illuminate the exact half-width of the specimen, where the camera was focused parallel to the oncoming droplets. Upon entering the laser sheet, the droplets were illuminated and visible as distinct features upon image acquisition. The finite thickness of the laser sheet further implied that very few unfocused droplets were observed.

\section{Experimental specimens}

A total number of 8 different materials referred to hereafter as specimens $\mathrm{A}-\mathrm{H}$ were utilized in this study in two different prescribed conditions; as supplied and in an eroded condition. The majority of these are established epoxy resins commonly used within the aerospace industry with addition of carbon nanotubes as a reinforcing agent. Specimens A and B are both combinations of Araldite ${ }^{\circledR}$ LY564/Aradur ${ }^{\circledR} 2954$ with the exception that specimen B also features multi-walled carbon nanotubes (MWCNT) at a $0.5 \%$ wt. Graphistrength ${ }^{\circledR}$ C100. Specimen $C$ is Araldite ${ }^{\circledR}$ DBF/Aradur ${ }^{\circledR}$ HY956EN base resin with $10 \%$ wt. aluminium nitride nanoparticles. Specimens $\mathrm{D}-\mathrm{G}$ are Araldite ${ }^{\circledR}$ MY0510/Aradur ${ }^{\circledR}$ 976-1 combinations where D and F have $0.5 \%$ wt. MWCNT. Finally specimen $\mathrm{H}$ is a gelcoat consisting of a SW404/ XB5173 combination. All considered material specimens have dimensions, $(L \times W)=(30 \times 20) \mathrm{mm}^{2}$ with different thickness values of $H \in[1,9] \mathrm{mm}$.

In the pristine state, no morphological treatment to the specimen surface is applied. This condition replicates instances where no material degradation has been observed upon implementation of the material on an aircraft during flight. The second state is an eroded state of the material, which is attained upon its wet blasting by using alumina. The choice of this abrasive stems from the need to obtain a fine degradation of the material without catastrophic failure. An eroded state of the material is obtained from an accelerated testing technique. However, this approach allows for establishment of surface morphology modifications on the resulting wetting characteristics of the materials. The surface roughness of the specimens prior and after wet blasting is measured by a Surtronic instrument. The average value of the surface roughness pre-erosion $R_{a}^{p}$, surface roughness posterosion $R_{a}^{e}$ and the percentage change in surface roughness $\Delta R_{a}$ are shown in Table 1 .

\subsection{Methodology}

The methodology comprises of determining the type of specimen based on its wetting characteristics, by employing the flowchart in Figure 2. 


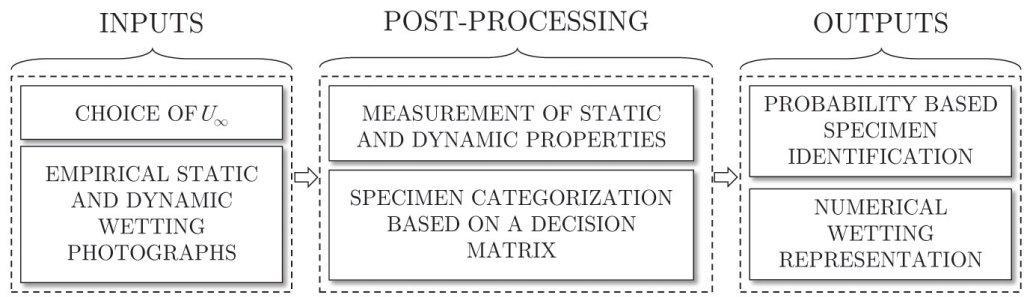

Figure 2: Flowchart for the data processing methodology based on empirical results.

Initially, a predefined free stream velocity $U_{\infty}$ at which the sample photographs are acquired is chosen by the user. This velocity determines the local liquid water content based on a numerical scheme that identifies the droplet cloud distribution from experimental photographs and provides an averaged histogram of the cloud spatial distribution on the target. Static and dynamic wetting photographs in the pre- and post-erosion state of the specimen are further used as inputs to the numerical specimen detection scheme. The resulting photographic information is upon input, processed based on a number of Graphical User Interfaced tools developed in MATLAB ${ }^{\circledR}$, as shown in Figure 3. Upon post-processing of the data, a decision matrix determines a probability factor which is attributed to a specific specimen.

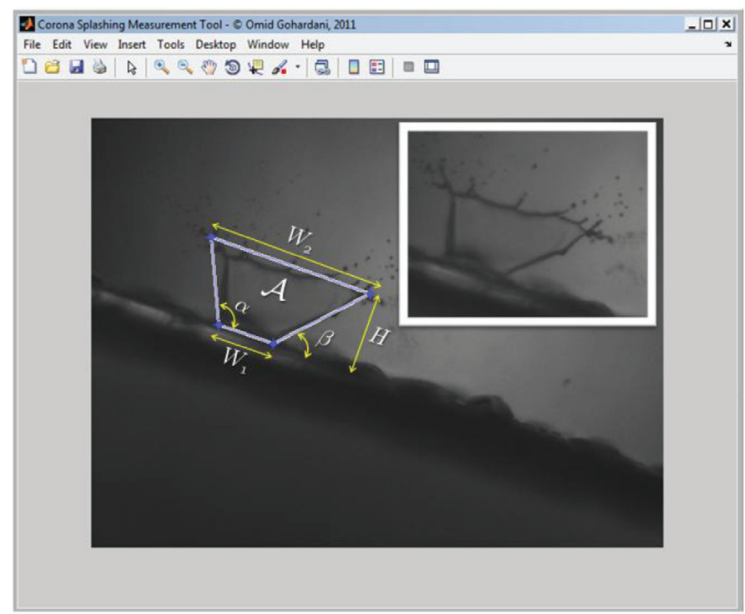

Figure 3: Autonomous corona measurement tool developed in MATLAB ${ }^{\circledR}$. The tool outputs the projected area of the corona $\mathcal{A}$, angles $\alpha$ and $\beta$, and distances $H, W_{1}, W_{2}$ and other image related properties. 


\section{Data acquisition}

\subsection{Liquid Water Content}

By utilizing a laser sheet for a given free stream velocity $U_{\infty}$, a sequential monitoring of the cloud scatter was actualized. The acquired photographs were digitally processed based a numerical approach which subdivided each photograph into 10 regions with the same size along the length of the specimen. The photographic stack of 347 images, was after contrast enhancement, processed by a droplet counting scheme which determined the number of droplets in each subregion. The averaged number of droplets within a sequence of images, in a given zone were further given by

$$
\bar{R}_{j}=\frac{1}{n}\left\{\sum_{i=1}^{n} D_{i}\right\}_{j}
$$

where $i=\{1,2, \ldots, n\}, j=\{1,2, \ldots, 10\}$ and $n$ is the number of frames equal to 347. Upon known number of droplets, a similar approach for estimation of the local Liquid Water Content $(L W C)$ as Ide [6] was employed. For a constant droplet diameter, $d$ and total sample area for the bins $\widetilde{A}$, the $L W C$ can be expressed as

$$
L W C \cong \frac{\rho \pi d^{3}}{6 \widetilde{A} U_{\infty} t} \sum_{i} R_{j}
$$

where $\rho$ is the water density, $t$ is the sampling time, $U_{\infty}$ is the free stream velocity, and the sum refers to the total number of droplets. The local liquid water content for free stream velocities $U_{\infty} \approx\{35,50,60\} \mathrm{m} \cdot \mathrm{s}^{-1}$ was estimated to $L W C \cong\{0.38,0.16,0.02\} \mathrm{g} \cdot \mathrm{m}^{-3}$. Figure 4 shows the averaged number of droplets within 10 equally sized bins at three different free stream velocities for a sampling duration of 10 seconds. The lack of droplets between the first and last bin for free stream velocities above $35 \mathrm{~m} \cdot \mathrm{s}^{-1}$ indicate that lower local $L W C$ values are observed with an increasing $U_{\infty}$. For statistical purposes $U_{\infty} \approx 35$ $\mathrm{m} \cdot \mathrm{s}^{-1}$ hence provides more reliable empirical results compared to the other free stream velocities.

\subsection{Contact angle measurements}

Both static and dynamic sessile drop methods were employed, in order to obtain angles at which the droplets move along the surface of the specimen. These methods convey results about the surface energy using Young's equation

$$
\gamma_{\mathrm{SG}}-\gamma_{\mathrm{SL}}-\gamma \cos (\varphi)=0
$$

where $\gamma$ is the surface energy and the subscripts SG and SL denote solid-gas and solid-liquid respectively. In the static sessile drop method, the angles between the 

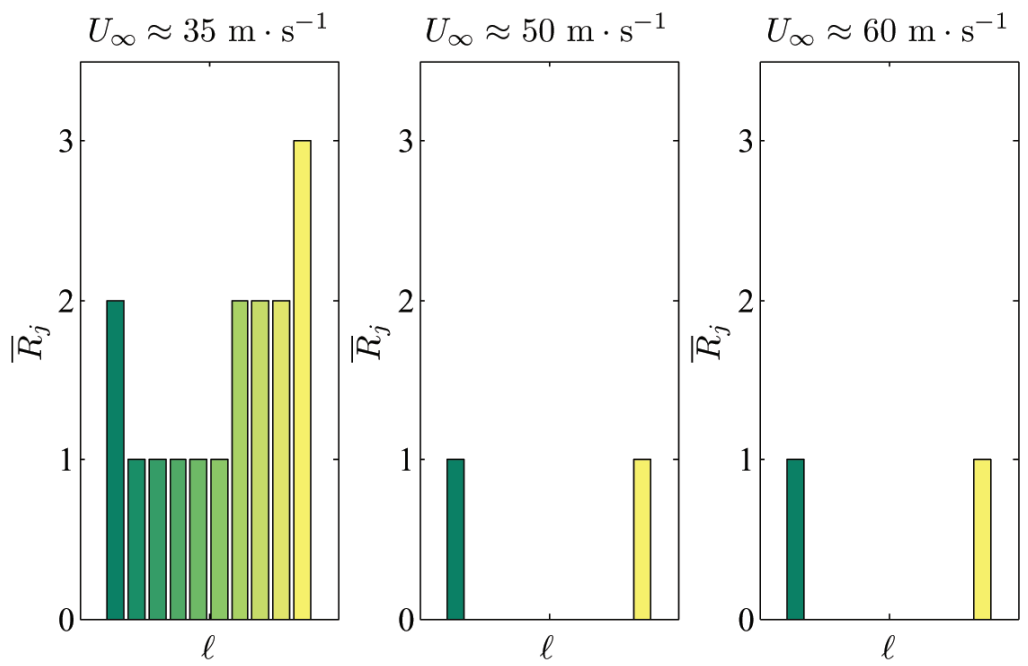

Figure 4: The number of averaged droplets within each bin $\bar{R}_{j}$, designated by a dark color for the first bin and a bright color for the last bin, for different free stream velocities $U_{\infty} \approx\{35,50,60\} \mathrm{m} \cdot \mathrm{s}^{-1}$ along the non-dimensional specimen length, $\ell$.

interfaces at SG and SL are measured by utilizing a protractor. For the dynamic sessile drop method, the drop is modified by either dispensing or retracting its volume, resulting in an advancing angle $\theta_{A}$ or a receding angle $\theta_{R}$, measured by a protractor. This method allows for an assessment of the homogeneity of the specimen, as droplets can be deposited on different locations on the specimen surface. In this study an averaged value of five measurements placed on different locations on the surface are presented. Hysteresis $\psi$, can in this context be defined as $\psi \equiv \theta_{A}-\theta_{R}$. The equilibrium Young contact angle $\theta_{C}$ can also be expressed in terms of the advancing and receding angles [8] as

$$
\theta_{C}=\arccos \left\{\frac{\Gamma_{A} \cos \left(\theta_{A}\right)+\Gamma_{R} \cos \left(\theta_{R}\right)}{\Gamma_{A}+\Gamma_{R}}\right\}
$$

where $\Gamma_{A, R} \equiv\left\{\sin ^{3}\left(\theta_{A, R}\right) /\left(2-3 \cos \left(\theta_{A, R}\right)+\cos ^{3}\left(\theta_{A, R}\right)\right)\right\}^{1 / 3}$. Table 1 , shows the advancing, receding, equilibrium Young contact angle and their corresponding hysteresis.

\subsection{Image analysis}

\subsubsection{Static wetting}

The static wetting of the specimens is assessed by employing contact angle measurements on both pristine and eroded specimen surfaces. The experimental setup for the contact angle measurements is shown in Figure 5. The specimen is 
Table 1: Surface roughness on the specimens in the pre- and post-erosion state given by $R_{a}^{p}$ and $R_{a}^{e}$ respectively. The percentage increase and decrease is given by $\Delta R_{a}=\left(R_{a}^{p}-R_{a}^{e}\right) / R_{a}^{p}$. The advancing angle $\theta_{A}$, receding angle $\theta_{R}$, hysteresis $\psi$ and the equilibrium Young angle $\theta_{C}$ for each specimen are shown with the pristine value in the left and the eroded value to the right in each column.

\begin{tabular}{|c|c|c|c|c|c|c|c|c|c|c|c|}
\hline \hline Notation & $R_{a}^{p}[\mu \mathrm{m}]$ & $R_{a}^{e}[\mu \mathrm{m}]$ & \multicolumn{2}{|c|}{$\Delta R_{a}$} & \multicolumn{2}{|c|}{$\theta_{A}\left[^{\circ}\right]$} & \multicolumn{2}{|c|}{$\theta_{R}\left[^{\circ}\right]$} & \multicolumn{2}{|c|}{$\psi\left[^{\circ}\right]$} & \multicolumn{2}{|c|}{$\theta_{C}\left[^{\circ}\right]$} \\
\hline $\mathrm{A}$ & 5 & 4.24 & -0.152 & 110 & 75 & 22 & 11 & 88 & 64 & 22 & 11 \\
$\mathrm{~B}$ & 0.74 & 4.4 & 4.946 & 93 & 90 & 33 & 6 & 60 & 84 & 35 & 6 \\
$\mathrm{C}$ & 0.8 & 3.74 & 4.946 & 80 & 90 & 17 & 10 & 64 & 80 & 18 & 10 \\
$\mathrm{D}$ & 0.2 & 3.98 & 3.675 & 87 & 80 & 28 & 10 & 58 & 70 & 30 & 10 \\
$\mathrm{E}$ & 1.2 & 3.92 & 2.267 & 93 & 70 & 32 & 8 & 62 & 62 & 33 & 8 \\
$\mathrm{~F}$ & 0.2 & 4.06 & 19.30 & 90 & 68 & 28 & 13 & 62 & 55 & 30 & 14 \\
$\mathrm{G}$ & 0.8 & 2.32 & 1.90 & 82 & 110 & 32 & 22 & 50 & 88 & 34 & 22 \\
$\mathrm{H}$ & 0.6 & 2.98 & 3.967 & 117 & 90 & 60 & 8 & 57 & 82 & 63 & 8 \\
\hline \hline
\end{tabular}

positioned on a table with a vertical adjustment capability and illuminated by a light emitting diode with a diffuser, which provides a uniform illumination of the droplet.

The setup is utilized for measurement of the advancing and receding contact angles on the different specimen surfaces.

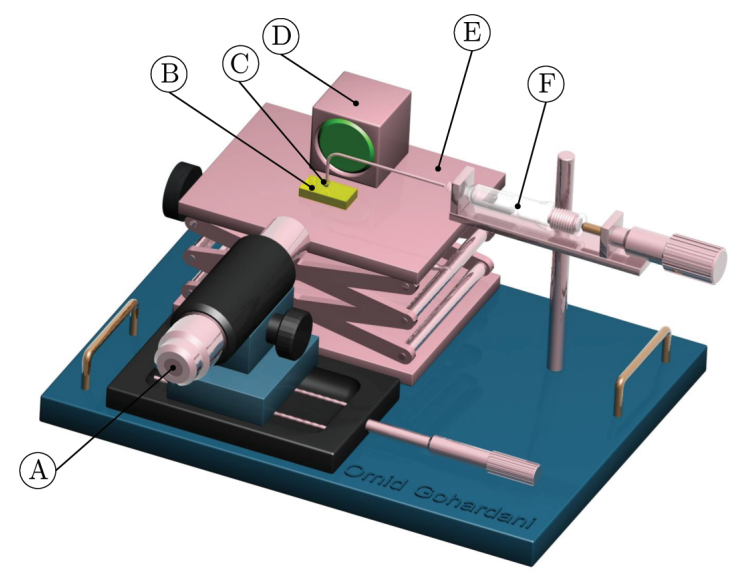

Figure 5: The experimental setup for the contact angle measurements, with notations: (A) Eye piece fitted with a protractor, (B) specimen, (C) generated droplet, (D) LED with diffuser, (E) vertical adjustable table, and (F) syringe and spring device fitted with a micrometer. 
Table 2: Non-dimensional parameters for the prescribed free stream velocities, $U_{\infty}$ in $\mathrm{m} \cdot \mathrm{s}^{-1}$.

\begin{tabular}{|c|c|c|c|c|c|}
\hline \hline$U_{\infty}$ & $R e$ & $W e$ & $K$ & $C a$ & $\xi^{*}$ \\
\hline 35 & 12271 & 6725 & 49856 & 0.55 & $3.03-3.04$ \\
50 & 17530 & 13725 & 101747 & 0.78 & 3.32 \\
60 & 21035 & 19764 & 146567 & 0.94 & 3.47 \\
\hline \hline
\end{tabular}

\subsubsection{Dynamic wetting}

The splashing phenomenon is often related to different non-dimensional parameters such as the Reynolds number $R e \equiv\left(\rho U_{\infty} d\right) / \mu$, the Weber number $W e \equiv\left(\rho U_{\infty}^{2} d\right) / \sigma$, the Ohnsorge number $O h \equiv \sqrt{W e} / R e$, and the Capillary number $C a \equiv W e / R e$, where $d$ is the droplet diameter, $\rho$ defines the density, $U_{\infty}$ is the free stream velocity, $\mu$ is the dynamic viscosity and $\sigma$ denotes the surface tension. The spreading factor $\xi^{*} \equiv d_{\max } / d$, can further be expressed as [7]

$$
\xi^{*}=\sqrt{\frac{W e+12}{3\left(1-\cos \left(\theta_{A}\right)+\frac{4 W e}{\sqrt{R e}}\right)}}
$$

Table 2, shows the non-dimensional parameters for the considered free stream velocities.

It is notable that the influence of the advancing angle on the spreading factor is nominal for the considered cases as it influences the maximum spread diameter, $d_{\max }$ with less than $5 \%$. From Table 2 , it is evident that $d_{\max } \in[3.0 d, 3.5 d]$ where $d$, is the initial diameter of the droplet. Representative photographs for each specimen in its pristine and eroded state respectively, are shown in Figure 6.

\section{Discussion}

The general observation for the wetting characteristics of the considered materials exhibits that specimens in the pre-eroded state are mainly hydrophobic as indicated by the formation of discrete water beads upon wetting. With modifications to the surface morphology by erosion, a partial or complete water film is apparent on all specimens.

The difference between the specimens in the post-eroded state is therefore, solely determined by the number of streams along the specimen surface. For the specimens exhibiting completely continuous water films, these streams merge to one coherent water film flowing along the specimen surface. By contrast, specimens with partial wetting characteristics have a discrete number of streams separated by non-wetted regions between them. 

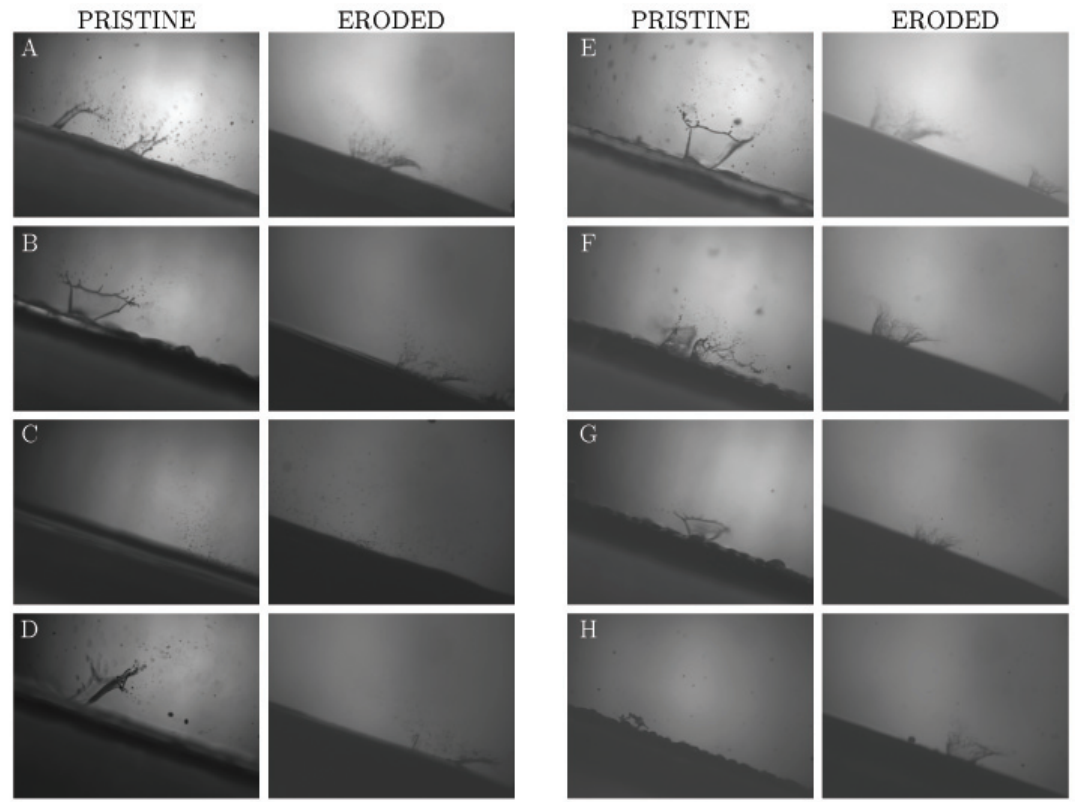

Figure 6: Pre- and post-erosion photographs of splashing structures on specimens $\mathrm{A}-\mathrm{H}$. The brightness and contrast of the images have digitally been enhanced for visualization purposes.

\section{Conclusions}

Dynamic wettability of the considered materials exhibit seemingly similar wetting characteristics in the pristine state of each material, with the materials being largely hydrophobic. Upon erosion, the formation of water films on the specimen surfaces, indicate a more hydrophilic behavior. Despite the complexities of image processing for different static and dynamic wettability arrangements, a methodology has been developed that discriminates between different empirical photographs of hydrophobic and hydrophilic specimens based on a numerical scheme. This scheme has thus far provided satisfactory results based on postprocessing of the empirical photographs. For more adequate results however new modules have to be added to this methodology that would allow for better identification rules of different materials, based on their wetting characteristics.

\section{Acknowledgement}

The authors greatly acknowledge the financial support from the European Union for conducting this research as a part of multi-functional layers for safer aircraft composite structures (LAYSA) project, under the Seventh Framework Programme (FP7), including aeronautics. 


\section{References}

[1] Shalin RE. Polymer matrix composites. 1995; Springer, pp 440. ISBN 0412613301.

[2] Gohardani O. Impact of erosion testing aspects on current and future flight conditions. 2011; Progress in Aerospace Sciences. Volume 47, Issue 4, Elsevier, pp. 280-303. doi:10.1016/j.paerosci.2011.04.001.

[3] Neinhuis $\mathrm{C}$ and Barthlott W. Characterization and distribution of waterrepellent, self-cleaning plant surfaces. 1997; Ann Bot, 79(6): pp 667-677.

[4] Byun D, Hong J, Saputra, Ko JH, Lee YJ, Park HC, Byun BK, Lukes JR. 2009; Wetting Characteristics of Insect Wing Surfaces. J Bionic Eng, Vol 6(1), pp 63-70.

[5] Hu DL, Prakash M, Chan B, and Bush JWM. Water-walking devices. In; Animal locomotion. 2010; Springer. Eds: Taylor G, Triantafyllou MS, Tropea C, pp 350. ISBN 3642116329.

[6] Ide RF. Comparison of Liquid Water Content measurement techniques in an icing wind tunnel. 1999; NASA/TM-1999-209643.

[7] Pasandideh-Fard M, Qiao YM, Chandra S, and Mostaghimi J. Capillary effects during droplet impact on a solid surface. 1996; Phys Fluids 8(3), pp 650-659.

[8] Tadmor R. Line Energy and the Relation between Advancing, Receding, and Young Contact Angles. 2004; Langmuir 20(18), pp 7659-7664. 